\title{
Investigation of robotic-assisted tilt-table therapy for early-stage spinal cord injury rehabilitation
}

\author{
Colm T. D. Craven, MEngSc; ${ }^{1-2 *}$ Henrik Gollee, PhD; ${ }^{1-2}$ Sylvie Coupaud, PhD; $;^{1-2}$ Mariel A. Purcell, MRCGP; ${ }^{2-3}$ \\ David B. Allan, FRCS ${ }^{2-3}$ \\ ${ }^{1}$ Centre for Rehabilitation Engineering, School of Engineering, University of Glasgow, Glasgow, UK; ${ }^{2}$ Scottish Centre \\ for Innovation in Spinal Cord Injury, Glasgow, UK; ${ }^{3}$ Queen Elizabeth National Spinal Injuries Unit, Southern General \\ Hospital, Glasgow, UK
}

\begin{abstract}
Damage to the spinal cord compromises motor function and sensation below the level of injury, resulting in paralysis and progressive secondary health complications. Inactivity and reduced energy requirements result in reduced cardiopulmonary fitness and an increased risk of coronary heart disease and cardiovascular complications. These risks may be minimized through regular physical activity. It is proposed that such activity should begin at the earliest possible time point after injury, before extensive neuromuscular degeneration has occurred. Robotic-assisted tilt-table therapy may be used during early-stage spinal cord injury (SCI) to facilitate stepping training, before orthostatic stability has been achieved. This study investigates whether such a stimulus may be used to maintain pulmonary and coronary health by describing the acute responses of patients with early-stage $(<1 \mathrm{yr})$ motor-complete SCI (cSCI) and motor-incomplete SCI (iSCI) to passive, active, and electrically stimulated robotic-assisted stepping. Active participation was found to elicit an increased response from iSCI patients. The addition of electrical stimulation did not consistently elicit further increases. Extensive muscle atrophy was found to have occurred in those patients with cSCI, thereby limiting the potential effectiveness of electrical stimulation. Active participation in robotic-assisted tilttable therapy may be used to improve cardiopulmonary fitness in iSCI patients if implemented as part of a regular training program.
\end{abstract}

Key words: cardiopulmonary fitness, functional electrical stimulation, lower limb, orthostatic hypotension, pulmonary response, rehabilitation, robotic assistance, spinal cord injury, tilt table, ventilatory response.

\section{INTRODUCTION}

Physical inactivity is associated with obesity, diabetes, and hypertension and is a significant cause for the development of coronary heart disease and cardiovascular attack [1]. These risks may be reduced through planned exercise and activity in the nondisabled population [2]. People with spinal cord injury (SCI) have neurological impairment, which can result in severe disability and progressive secondary health complications to the cardiopulmonary and musculoskeletal systems. Their life expectancy is less than that of the general population [3], and they are at a higher risk of rehospitalization and premature death because of cardiovascular disease and diabetes mellitus [4-7]. Rehospitalization results in a considerable burden on the healthcare system, emotional trauma, and reduced quality of life $[6,8]$. People with SCI lead sedentary lifestyles as a consequence

\footnotetext{
Abbreviations: $\mathrm{cSCI}=$ motor-complete SCI, FES $=$ functional electrical stimulation, iSCI $=$ motor-incomplete SCI, MAP $=$ mean arterial blood pressure, MET $=$ metabolic equivalent, RATTT $=$ robotic-assisted tilt-table therapy, RER $=$ respiratory exchange ratio, $\mathrm{SCI}=$ spinal cord injury.

*Address all correspondence to Colm T. D. Craven, MEngSc; Centre for Rehabilitation Engineering, School of Engineering, James Watt (South) Building, University of Glasgow, Glasgow G12 8QQ, UK; +44-141-330-4406.

Email: c.craven.1@research.gla.ac.uk. http://dx.doi.org/10.1682/JRRD.2012.02.0027
} 
of their injuries, and their functional capabilities are less than those of their counterparts in the general population.

In general, inactivity has been shown to result in a rapid decline in aerobic fitness of 0.8 to 0.9 percent per day during prolonged periods of bed rest [9]. Exercise capacity and functional ability in SCI are related to the neurological level and severity of cord injury [1,10-11]. Muscle paralysis causes rapid and extensive muscle atrophy, which reduces daily energy requirements and leads to a decline in aerobic fitness. The inability to use large and highly oxidative muscles, such as those in the lower limbs, reduces the ability to achieve a high metabolic demand during exercise. In addition, individuals with SCI exhibit a diminished response to exercise, during which hypokinesis [12], hypotension, reduced cardiac output [13], blunted blood pressure [14], and slow oxygen uptake kinetics [15] have been found.

Arm crank ergometry is the most commonly found and readily accessible form of exercise available to those with SCI. Peak power outputs between 10 and $100 \mathrm{~W}$ can be achieved during arm crank ergometry, depending on the neurological level and severity of injury [1]. However, the low metabolic demand of the relatively small muscle mass of the upper limb and the increased risk of shoulder pain limit effectiveness and use of arm crank ergometry [16]. Peak oxygen uptake levels achieved by nondisabled, neurologically intact test subjects during arm crank ergometry have been found to be 70 percent of those achieved during maximal treadmill testing [1]. Alternative assistive technologies that facilitate exercise using the lower limbs include robotic-assisted treadmill exercise and functional electrical stimulation (FES)assisted leg-cycle ergometry. Active participation during robotic-assisted treadmill exercise has been found to elicit an increased cardiovascular response from SCI subjects [17], and FES-assisted leg-cycle ergometry has been shown to improve cardiovascular fitness and exercise tolerance [15,18-19]. Peak power outputs in untrained people with SCI have been found to be between 10 and $13 \mathrm{~W}$ during FES-assisted leg-cycle ergometry [20-22] and between 12 and $48 \mathrm{~W}$ during robotic-assisted treadmill exercise [17]. These technologies are normally employed in a sitting or standing posture when orthostatic tolerance has been achieved and are thus typically used in the chronic ( $>6 \mathrm{mo}$ ) phases of SCI rehabilitation.

Orthostatic hypotension is common in the early period of SCI and affects the subjects' ability to exercise. Intractable orthostatic hypotension can delay early mobilization and participation in the rehabilitation activities described previously. It is characterized by light-headedness, nausea, weakness, palpitations, and syncope and is caused by a rapid drop in blood pressure during head-up tilt [23]. In SCI, vasodilation results in the peripheral pooling of blood, which compromises venous return and reduces cardiac output. This phenomenon has been reported in a tilt-table study that found blood pressure, as recorded from individuals with early-stage $\mathrm{SCI}$, to be inversely proportional to increasing tilt angle from the horizontal position [24].

Tilt-table therapy is commonly used to treat orthostatic hypotension. During tilt-table therapy, an individual will be gradually accustomed to head-up tilt by incrementally increasing tilt angle over a period of time. It has been found that tilt-table therapy can be enhanced by the application of FES to the knee extensors and plantar flexors [24]. The improved orthostatic tolerance found was attributed to increased peripheral resistance caused by the muscle contractions elicited by FES. In a similar investigation with nondisabled adults, passive movement of the lower limbs was found to maintain orthostatic stability during robotic-assisted tilt-table therapy (RATTT) [25]. Though different in application, it was concluded that the pumping action of the lower limbs during RATTT is analogous to skeletal muscle pump. In both instances, orthostatic instability is counteracted by increased peripheral resistance to the gravitational pooling of blood. A study investigating RATTT augmented by FES concluded that the combination of these therapies was more effective in improving orthostatic tolerance than either intervention individually [26-27].

In addition to improving orthostatic tolerance, RATTT may also be an effective exercise tool. Initiating the rehabilitation process at the earliest possible time point after SCI may minimize losses in aerobic fitness that arise because of inactivity. It is hypothesized that RATTT may be used to provide a strong training stimulus to complement conventional physiotherapy practices and serve the dual purpose of increasing orthostatic tolerance and attenuating the decline in aerobic fitness. The metabolic demand exerted during passive, active, and FES-assisted stepping during RATTT has not yet been investigated in people with SCI. This article describes a cross-sectional pilot study investigating the physical exertion associated with early-stage $(<1 \mathrm{yr})$ SCI. Neurologically and orthopedically stable, nonacute $(>1 \mathrm{mo})$ SCI patients were recruited to first determine the validity of our hypothesis and to investigate the potential of RATTT for improved 
aerobic fitness. The aim of this study is twofold: to investigate the physical exertion of RATTT by describing the ventilatory and cardiopulmonary response to passive, active, and FES-assisted RATTT; and to investigate the difference in response of patients with motor-complete $\mathrm{SCI}(\mathrm{cSCI})$ and motor-incomplete SCI (iSCI).

\section{METHODS}

\section{Subjects}

Three cSCI and three iSCI participants were recruited from the Queen Elizabeth National Spinal Injuries Unit, Glasgow, United Kingdom (Table 1). The main inclusion criteria were patients with paraplegia or tetraplegia due to a spinal cord lesion with no lower motor neuron damage.

\section{Robotic-Assisted Tilt-Table}

RATTT uses a tilt-table with an integrated roboticassisted stepping device (Figure 1). It integrates a standard tilt-table with robotic orthoses that impose a trajectory on the lower limbs in a manner that approaches nondisabled, physiological hip kinematics. An upperbody harness secures the subject to the table and provides support around the chest and abdomen. The table can be tilted in a range from $0^{\circ}$ to $80^{\circ}$ from the horizontal position. Linearly driven robotic orthoses are attached to the thighs, and the knee joint is passively moved to full flexion and full extension to establish range of motion. During stepping, the robotic orthoses automatically impose a stepping trajectory on the lower limbs in a manner such that a flexion/extension movement profile is achieved at the hip and knee joints within this preset range. The guidance force of the robotic orthoses can be adjusted to match the functional ability of the patient. Patients with a cSCI are provided with full guidance, whereas those with iSCI may be provided with reduced guidance to encourage volitional effort. The stepping rate imposed by the robotic orthoses can be set within a range of 4 to 40 steps/ min for each leg.

The feet are secured to foot plates that have an integrated spring system. The springs become loaded during hip/knee extension and therefore provide resistance to movement. The springs release during hip/knee flexion. Each individual spring has a calculated spring constant of $9 \mathrm{kN} / \mathrm{m}$ and can be extended up to a maximum displacement of $0.05 \mathrm{~m}$ during hip/knee extension. The work input per step $(W)$ required to fully extend each spring is assumed to be equal to the potential energy stored within the spring at this displacement and is calculated to be 11.25 J using Equation (1).

$$
W=\frac{1}{2} k x^{2},
$$

where $k=$ spring constant and $x=$ displacement.

Work rate during RATTT can thus be estimated using Equation (2).

$$
\dot{W}=W \dot{s},
$$

where $\dot{W}=$ work rate and $\dot{S}=$ stepping rate.

FES may be used to augment stepping during RATTT and is applied using a biphasic, current-controlled electrical stimulation device (Motionstim, Krauth + Timmermann, $\mathrm{GmbH}$; Hamburg, Germany). Stimulation is delivered via transcutaneous neuromuscular electrical stimulation electrodes (PALS Platinum, Axelgaard; Lystrup, Denmark) placed over the quadriceps $(5 \times 10 \mathrm{~cm}$ oval $)$, hamstring $(5 \times$

Table 1.

\begin{tabular}{|c|c|c|c|c|c|c|}
\hline Subject & Lesion & Level & Age (yr) & Weight (kg) & Time Postinjury (wk) & AIS \\
\hline$\overline{\mathrm{S} 1}$ & $\mathrm{cSCI}$ & T4 & 22 & 70 & 16 & $\mathrm{~A}$ \\
\hline S3 & $\mathrm{cSCI}$ & $\mathrm{C} 3$ & 25 & 60 & 21 & $\mathrm{~B}$ \\
\hline S4 & iSCI & T9 & 53 & 86 & 46 & $\mathrm{C}$ \\
\hline S5 & iSCI & $\mathrm{T} 2$ & 44 & 98 & 40 & $\mathrm{D}$ \\
\hline
\end{tabular}

Subject characteristics. 


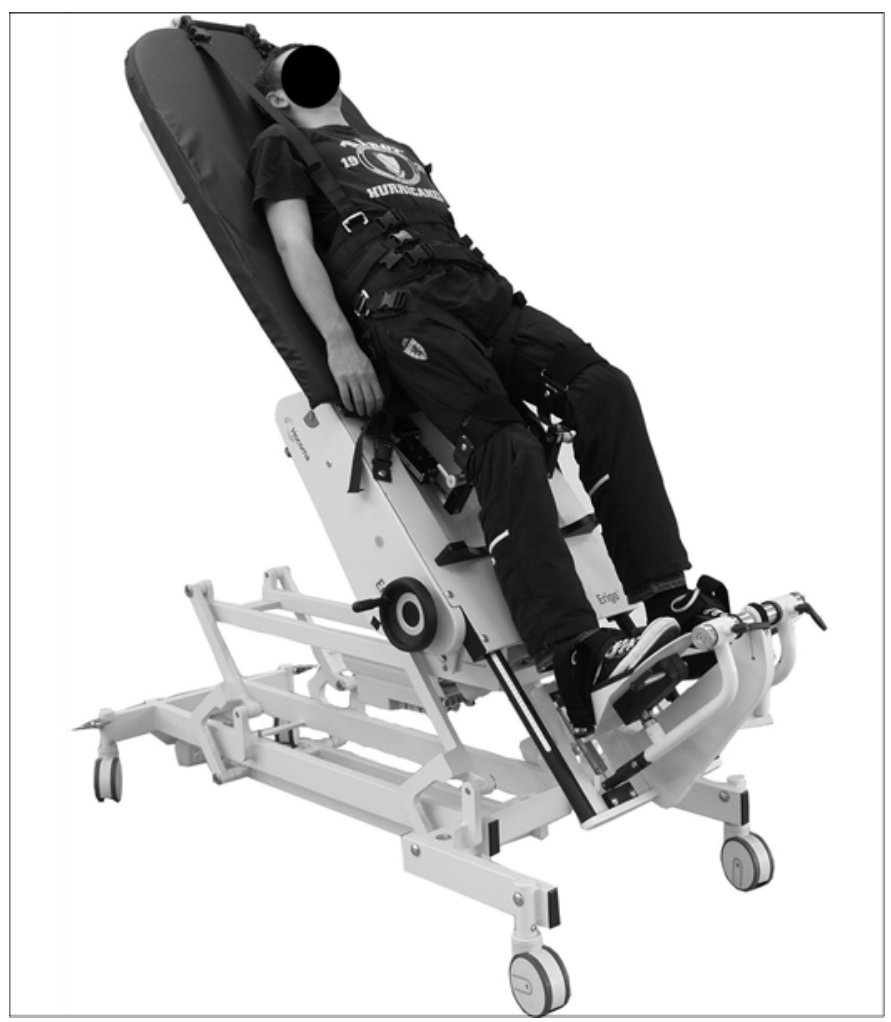

\section{Figure 1.}

Tilt-table with integrated robotic-assisted stepping device (Erigo, Hocoma AG; Switzerland).

$10 \mathrm{~cm}$ oval $)$, and calf $(5 \times 6.4 \mathrm{~cm}$ oval $)$ muscle groups. Stimulation frequency and pulse width are fixed at $20 \mathrm{~Hz}$ and $300 \mu$ s, respectively. Stimulation current is adjustable for each individual muscle group. Stimulation timing is automatically synchronized during stepping by the RATTT device via integrated position sensors that continuously measure the angular positions of the robotic orthoses. In this way, we know when flexion/extension of the hip/knee joints occurs during stepping. The hamstring and calf muscle groups are stimulated during knee flexion, and the quadriceps muscle group is stimulated during knee extension.

\section{Real-Time Feedback}

The angular positions of the robotic orthoses were sampled at $50 \mathrm{~Hz}$ using a USB-6009 analog-to-digital data acquisition card (National Instruments Corp; Austin, Texas), recorded on a laptop personal computer, and displayed in real-time to the subject using a custom-built interface in LabVIEW (National Instruments Corp). The interface displayed the preset range of motion and a red bar indicating the current angular position of the orthoses. The bar changed to green if the subject achieved a range of motion during stepping that was within 5 percent of the preset range. Where appropriate (cf testing protocol), subjects were encouraged to increase their volitional effort to maintain full range of motion as indicated by the real-time feedback.

\section{Measurement Equipment}

Pulmonary gas exchange and ventilatory measurements were performed using a breath-by-breath analysis system (MetaMax 3B, Cortex Biophysic GmbH; Leipzig, Germany) and recorded on a laptop computer. Prior to use, we calibrated the system by performing a volume analysis using a $3 \mathrm{~L}$ volumetric syringe; gas calibration was carried out using ambient air and a certified precision-analyzed gas mixture. Heart rate was measured and recorded using a short-range telemetry heart rate monitor (Polar S410, Polar Electro Oy; Kempele, Finland). Blood pressure measurements were performed using an automatic blood pressure monitoring device (M7, Omron Healthcare Europe BV; Hoofddorp, the Netherlands).

\section{Testing Protocol}

Each subject participated in three experimental sessions. During the first session, the subject was familiarized with the device and all measurement equipment. Testing was carried out in the two subsequent experimental sessions. The subject was asked not to perform any strenuous exercise or consume alcohol in the $24 \mathrm{~h}$ prior to testing, not to ingest any caffeine in the $4 \mathrm{~h}$, and not to eat in the $2 \mathrm{~h}$ prior to a testing session. No two experimental sessions were carried out on consecutive days. The subjects wore similar clothing and were in good health for each session.

During a testing session, the subject was first fitted with the heart rate monitor and secured to the RATTT device using the upper-body harness. Transcutaneous neuromuscular electrical stimulation electrodes were then placed over the quadriceps, hamstring, and calf muscle groups while the subject was in a supine position. Stimulation was then applied, and the current was increased (28-100 mA) for each individual muscle group. For subjects with no sensation, stimulation currents were set at a level at which a maximum palpable contraction (that did not induce spasm) could be detected. For those subjects with remaining sensation, stimulation currents were set at a level at which a maximum palpable 
contraction (that was also comfortable) could be detected. The subject's legs were secured to the linearly driven orthoses and foot plates, and full range of motion was established. A cuff was then placed above the elbow of the left arm to allow blood pressure measurement from the brachial artery. The subject was finally fitted with the mask for the breath-by-breath analysis system.

The testing protocol consisted of five discrete phases, which were carried out once in each experimental session. Each phase was $5 \mathrm{~min}$ in duration. Pulmonary gas exchange, ventilator, and heart rate measurements were continuously recorded throughout the course of a testing session. Discrete blood pressure measurements were taken in the final $30 \mathrm{~s}$ of each phase.

Descriptions of the five phases of the testing protocol follow: Phase 1: the subject was in supine posture and no stepping profile was imposed by the robotic orthoses. Phase 2: the subject was tilted to an angle of $70^{\circ}$ from horizontal position at an angular velocity of $0.06 \mathrm{rad} / \mathrm{s}$. Phase 3: the robotic orthoses were activated and set to provide full guidance force. iSCI subjects were instructed not to exert any volitional effort during this phase. Phase 4a (iSCI only): the guidance force provided by the robotic orthoses was reduced, and the subject was instructed to compensate by increasing their volitional effort. Guidance force was set at a level that the subject was comfortable with in a range of 50 to 90 percent of maximum. Volitional effort was ensured using the realtime feedback. Phase 4b: FES was applied to augment subject's participation in the stepping cycle. In addition to the application of FES, the iSCI subjects were instructed to continue volitional participation in the stepping cycle.

During stepping, the subjects worked against the resistance provided by the integrated spring system found at the foot plates. Stepping was set at a rate that each individual subject was comfortable with, which was found to be between 10 and $20 \mathrm{steps} / \mathrm{min}$. At these stepping rates, the work rate of each leg during stepping was calculated to be between 1.8 and $3.7 \mathrm{~W}$.

\section{Outcome Measures}

The outcome measures were oxygen uptake, respiratory exchange ratio (RER), minute ventilation, heart rate, and mean arterial blood pressure (MAP).

\section{Breath-by-Breath Analysis}

Oxygen uptake, RER, and minute ventilation were processed using the MATLAB Signal Processing Toolbox (MATLAB, The MathWorks Inc; Natick, Massachusetts). Oxygen uptake was normalized to body weight, and all data were smoothed using a tenth-order, movingaverage filter and discretized according to each phase of the testing protocol. The maximum value in the final $90 \mathrm{~s}$ of each phase (deemed to represent "steady-state" gas exchange for the phase) was then found and averaged, for each subject respectively, over the first and second testing sessions.

\section{Heart Rate}

Heart rate was normalized to each subject's target heart rate using Equation (3) $[1,28]$ and otherwise analyzed according to the methods described for the breathby-breath data.

$$
\mathrm{HR}_{\% \max } \cong \frac{\mathrm{HR}}{220-\text { age }},
$$

where $\mathrm{HR}_{\% \text { max }}=$ heart rate as a percentage of the target heart rate, and $\mathrm{HR}=$ recorded heart rate.

\section{Mean Arterial Blood Pressure}

MAP was calculated from the discrete blood pressure measurements using Equation (4) [29].

$$
\text { MAP }=\frac{P_{\text {sys }}-P_{\text {dia }}}{3}+P_{\text {dia }}
$$

where $P_{\text {sys }}=$ systolic blood pressure and $P_{\text {dia }}=$ diastolic blood pressure.

\section{Statistical Analysis}

Statistical analysis was performed using the MATLAB Statistics Toolbox. Data normality was assessed using quantile-quantile plots, and a Levene test was used to check for equal variance. A repeated measures analysis of variance and post-hoc multiple comparisons procedure with Bonferroni correction were used to investigate the response of the iSCI and cSCI subjects, respectively, to each phase of the testing protocol. Two-sample $t$-tests were used to investigate the difference in response of the iSCI and cSCI subjects for a given phase of testing. Pearson correlation coefficients were used to investigate the test-retest reliability of the obtained outcome measures. 
Cohen's $d$, based on an average standard deviation, was used to estimate effect size.

\section{RESULTS}

All subjects completed each of the experimental sessions. Head-up tilt was tolerated well, and no instances of hypotension or autonomic dysreflexia occurred. None of the subjects had significant spasticity. In one instance, the application of FES to the calf muscle group of Subject 1 was limited because of the elicitation of spasm in the homonymous muscle during stimulation, although this did not affect the trajectory of the lower limb during robotic-assisted stepping. The oxygen uptake, RER, minute ventilation, and heart rate response of each subject are shown in Figure 2.

For the subjects with iSCI, we found that oxygen uptake, RER, minute ventilation, and heart rate were (a)

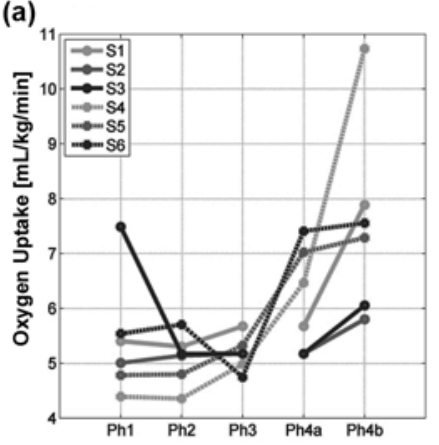

(c)

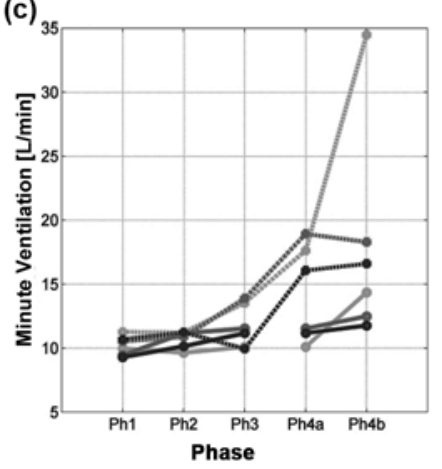

(b)

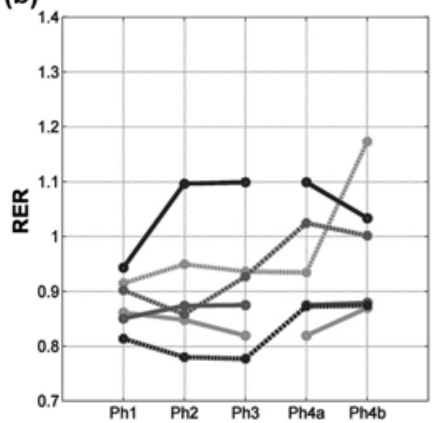

(d)

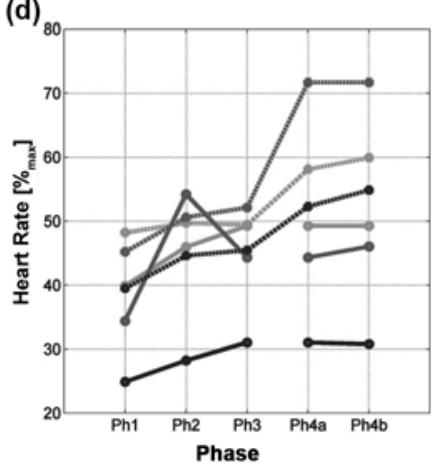

Figure 2.

Plot of results for each individual subject (S), which include (a) oxygen uptake, (b) respiratory exchange ratio (RER), (c) minute ventilation, and (d) heart rate. Ph1-Ph4b: phase of testing. Motor-complete spinal cord injured subjects (S1-S3) did not undergo Phase $4 \mathrm{a}$ of testing protocol, which involved volitional contribution to robotic-assisted stepping cycle. Max = maximum. unchanged throughout the first three phases (Phase 1-3) of the testing protocol (Table 2). Volitional participation (Phase 4a) in the stepping cycle and the addition of FES (Phase $4 \mathrm{~b}$ ) led to an increase in oxygen uptake, RER, minute ventilation, and heart rate, and these increases were found to be significant. No statistically significant change in MAP was found throughout the testing protocol. For the cSCI subjects, a small and statistically significant increase in minute ventilation was identified, but no change in the other outcome measures was found. The results of the statistical analysis are summarized in Table 2.

Pearson correlation coefficients indicated a high degree of test-retest reliability for both the iSCI and cSCI subjects. Correlation coefficients were found to be between 0.64 and 0.95 and were significant at $p<0.05$, implying that there is a large or very large between-test relationship for each of the outcome measures.

Comparing the iSCI and cSCI subjects, no difference in RER, minute ventilation, or heart rate response between either group was found throughout the first three phases (Phase 1-3) of testing. During Phase 4b, we found that the oxygen uptake, minute ventilation, and heart rate of the iSCI subjects were significantly larger than those of the cSCI subjects in the same phase. The significantly larger responses found for the iSCI subjects is attributed to the large effect, as computed using Cohen's $d$, of volitional participation during this phase. Cohen's $d$ was calculated to be 1.32 (oxygen uptake), 1.49 (minute ventilation), and 2.22 (heart rate). MAP was found to be significantly larger across all phases for the iSCI subjects than for the cSCI subjects.

\section{DISCUSSION}

This cross-sectional pilot study investigates the feasibility of RATTT as a potential exercise therapy. We hypothesized that this technique may be employed during the very early stages of SCI rehabilitation to facilitate cardiopulmonary exercise. It may be of most benefit during the acute stage of injury, before orthostatic tolerance is achieved and more traditional exercise techniques have commenced. The participants in this study were within their first year of injury and were recruited because they were orthopedically and neurologically stable. These subjects would be considered to be early-stage rather than acute patients. 
Table 2.

Mean oxygen uptake, respiratory exchange ratio (RER), minute ventilation, heart rate, and mean arterial blood pressure for incomplete and complete spinal cord injured subjects (iSCI and cSCI, respectively) are presented for each phase of experimental protocol.

\begin{tabular}{|c|c|c|c|c|c|c|c|c|}
\hline Parameter & Injury & $\begin{array}{c}\text { Phase } 1 \\
\text { Mean } \pm \text { SD }\end{array}$ & $\begin{array}{c}\text { Phase } 2 \\
\text { Mean } \pm \text { SD }\end{array}$ & $\begin{array}{c}\text { Phase } 3 \\
\text { Mean } \pm \text { SD }\end{array}$ & $\begin{array}{c}\text { Phase 4a } \\
\text { Mean } \pm \text { SD }\end{array}$ & $\begin{array}{c}\text { Phase 4b } \\
\text { Mean } \pm \text { SD }\end{array}$ & $\begin{array}{c}\text { RM } \\
F(d f) \\
\end{array}$ & $p$-Value \\
\hline$(\mathrm{mL} / \mathrm{kg} / \mathrm{min})$ & $\mathrm{cSCI}$ & $5.96 \pm 1.35$ & $5.20 \pm 0.57$ & $5.34 \pm 0.28$ & - & $6.58 \pm 1.06$ & $F(3)=1.48$ & 0.31 \\
\hline & cSCI & $0.89 \pm 0.06$ & $0.94 \pm 0.13$ & $0.93 \pm 0.14$ & - & $0.93 \pm 0.09$ & $F(3)=0.84$ & 0.52 \\
\hline Minute Ventilation & iSCI & $10.82 \pm 0.64$ & $11.10 \pm 0.67$ & $12.45 \pm 2.33$ & $17.54 \pm 3.01$ & $23.12 \pm 9.65$ & $F(4)=4.41$ & 0.04 \\
\hline$(\mathrm{L} / \mathrm{min})$ & $\mathrm{cSCI}$ & $9.57 \pm 0.45$ & $10.31 \pm 1.28$ & $10.93 \pm 0.91$ & - & $12.86 \pm 1.43$ & $F(3)=6.62$ & 0.02 \\
\hline Arterial Pressure & iSCI & $104.17 \pm 18.82$ & $108.17 \pm 20.73$ & $118.17 \pm 23.53$ & $110.67 \pm 25.55$ & $114.83 \pm 16.69$ & $F(4)=1.05$ & 0.44 \\
\hline$(\mathrm{mm} \mathrm{Hg})$ & cSCI & $86.78 \pm 2.47$ & $85.67 \pm 10.98$ & $84.78 \pm 13.45$ & - & $87.39 \pm 13.34$ & $F(3)=0.08$ & 0.97 \\
\hline
\end{tabular}

In agreement with previous studies [26-27], head-up tilt was well tolerated during the experiments, with no instances of presyncope or syncope occurring. Some patients, particularly those with inhibited motor function and compromised vasomotor control, would be considered to be at a high risk of orthostatic instability. When considered as a group, no significant changes in MAP were found during any phase of the testing protocol for the iSCI or cSCI subjects. The MAP of the iSCI group was found to be significantly higher than that of the cSCI group. This is most likely a consequence of the higher average age of the iSCI (50 yr) versus the cSCI (22 yr) group; systolic and diastolic blood pressure are generally expected to increase with age [30]. Alternatively, those with an iSCI may be exhibiting increased venous return in comparison to their cSCI counterparts because of the presence of an effective skeletal muscle pump, particularly during active movement of the lower limbs. Increased venous return would result in higher MAP as recorded at the brachial artery. Some short-duration spikes in MAP may have occurred during the transition from one testing condition to another, but the likelihood of such changes being detected is low because blood pressure was recorded only at discrete time intervals, which were at the end of each phase. Such spikes were found in the continuously recorded heart rate of two subjects (Subjects 1 and 2) at the beginning of head-up tilt (Phase 2) and at the initiation of robotic-assisted stepping (Phase 3). These sharp increases, however, soon dropped and stabilized at the reported values.

When considered together, the responses of the iSCI and cSCI groups are generally comparable throughout the first three phases (Phase 1-3) of the testing protocol. Small increases in minute ventilation, oxygen uptake, and heart rate were found during head-up tilt (Phase 2) and head-up tilt with passive robotic-assisted stepping (Phase 3). However, these increases were not found to be significant, and no significant difference between the iSCI and cSCI groups were detected.

Due to the nature of their paralysis, the cSCI subjects were unable to volitionally contribute to the roboticassisted stepping cycle (Phase 4a). Active participation during this phase by the iSCI subjects did elicit an increase in oxygen uptake, minute ventilation, and heart rate, and the increase in heart rate was found to be significant.

FES was used to augment the subjects' volitional contribution during the final phase of the testing protocol (Phase $4 \mathrm{~b}$ ). The cSCI participants progressed directly to this phase upon completing Phase 3. For the cSCI subjects, the increased cardiopulmonary and ventilatory responses were small, but significant in the case of minute ventilation. These increases, however, are unlikely to be of sufficient magnitude to lead to improved fitness if implemented in a training program. Larger increases in these parameters were found for the iSCI subjects, and in most cases, the increases were found to be significant. These results indicate that FES potentiates the cardiopulmonary and ventilatory response of iSCI subjects during RATTT. An inspection of the group averages reveals that volitional participation during FES-assisted RATTT results in the largest increases in cardiopulmonary and ventilatory response. Considering each subject individually, we found that the continued increases found are attributable to Subject 4 only. The responses from Subject 5 and 6 remain consistent throughout the last two phases of testing (Phase $4 a$ and $4 b$ ).

Functional ability and response to exercise are related to the neurological level and severity of cord 
injury [1]. Those with a lower-level injury are expected to have more functional muscle available with which physical activity can be performed. Investigations comparing individuals with incomplete tetraplegia, high paraplegia, and low paraplegia report that physical capacity for exercise decreases with increasing levels of cord injury [11]. Decreased capacity for exercise is manifested as a lower achievable peak oxygen uptake and lower peak heart rate. Therefore, in this study, we expected that those with a lower-level injury would perform better. In keeping with this assumption, we found that the subject with low paraplegia (Subject 4) performed considerably better than subjects with higher level injuries.

Oxygen uptake and RER are key indicators of the stress being exerted on the cardiopulmonary system during exercise. The test-retest reliability of these parameters was found to be high, and as such, they provide a reliable indication of the degree of physical exertion experienced during RATTT. Oxygen uptake is commonly expressed as a metabolic equivalent (MET), where 1 MET is equivalent to a normalized oxygen uptake value of $3.5 \mathrm{~mL} / \mathrm{kg} / \mathrm{min}$, which is the approximate resting metabolic rate of a typical $70 \mathrm{~kg}, 40 \mathrm{yr}$ old male [28]. For the iSCI subjects, normalized oxygen uptake was recorded to be between 5.3 and $11.0 \mathrm{~mL} / \mathrm{kg} / \mathrm{min}$ during Phase 4 of the testing protocol, which equates to 1.5 to 3.1 METs. This is defined by the World Health Organization to be low- to moderate-intensity physical activity. Moderate-intensity physical activity is defined as being between 3 and 6 METs, and high-intensity physical activity is carried out at 6 METs and above [31]. The peak oxygen uptake values found in this study are considerably lower than the maximum achievable values in agematched persons from the general population, which are expected to be in the range of 34 to $44 \mathrm{~mL} / \mathrm{kg} / \mathrm{min}$ [32]. However, such levels of oxygen uptake are difficult for people with SCI to achieve because of the inability to effectively recruit all available muscles. A more appropriate comparison is peak oxygen uptake in people with chronic SCI during wheelchair and leg cycle ergometry, where values of 11.14 to $21.6 \mathrm{~mL} / \mathrm{kg} / \mathrm{min}$ are typical $[21,33]$. In summary, the levels of oxygen uptake elicited in the iSCI subjects during volitional participation in RATTT approach the peak values expected of this patient population. In addition, RER did not usually exceed 1 , indicating that activity of this nature is predominantly aerobic and likely to be sustainable. These data suggest that volitional participation in iSCI may be used for low- to moderate-intensity physical activity, and if instituted regularly, this form of activity may be used to maintain cardiopulmonary fitness.

For the cSCI subjects, normalized oxygen uptake was found to be between 5.0 and $7.5 \mathrm{~mL} / \mathrm{kg} / \mathrm{min}$ during FES-assisted RATTT, which equates to 1.4 to 2.1 METs. In comparison, maximum oxygen uptake in age-matched individuals from the general population is expected to be between 43 and $52 \mathrm{~mL} / \mathrm{kg} / \mathrm{min}$ [32]. However, as with the iSCI subjects, these levels of oxygen uptake are unlikely to be attainable in those with cSCI. The peak oxygen uptake values recorded in this study are comparable to those reported for chronic, untrained cSCI individuals during FES-assisted leg cycle ergometry, where typical values were found to be $7.3 \pm 2 \mathrm{~mL} / \mathrm{kg} / \mathrm{min}$ [18]. These comparisons are considered to be most valid because FES-assisted leg cycle ergometry, like RATTT, uses the lower limb only, with little or no contribution from the upper limb. It is unlikely that FES-assisted RATTT sustained at this intensity will result in improved cardiopulmonary fitness as it would be necessary to maintain this level of intensity of physical activity for durations in excess of 150 min per week.

It is likely that the small cardiopulmonary responses reported here for the cSCI subjects are due to the small demand imposed by the muscles of the lower limbs, which had undergone extensive muscular decline. A period of FES-assisted leg cycle ergometry training has been shown to counteract this decline and lead to an improved cardiopulmonary response [18]. In FEScycling studies, typical peak oxygen uptake values after training of $11.7 \pm 3.2 \mathrm{~mL} / \mathrm{kg} / \mathrm{min}$ have been reported. We would thus expect that a period of FES-assisted RATTT training would result in a similar increment in peak oxygen uptake and, therefore, lead to an improved cardiopulmonary response.

When comparing the cSCI and iSCI subjects during FES-assisted RATTT (Phase 4b), we found that oxygen uptake, minute ventilation, and heart rate were significantly larger for the iSCI subjects. The computed effect size for each of these parameters was found to be large, indicating that type of injury, and by extension the ability to volitionally contribute to the stepping cycle, largely accounts for the difference in response found. These data suggest that volitional participation is key in eliciting an increased cardiopulmonary and ventilatory response in participants during RATTT. 
This study assessed a small sample of subjects. The average age, weight, and time since injury of the cSCI group ( $22 \mathrm{yr}, 67 \mathrm{~kg}$, and $18 \mathrm{wk}$, respectively) were considerably different from those of the iSCI group $(50 \mathrm{yr}$, $88 \mathrm{~kg}$, and $32 \mathrm{wk}$, respectively). Extensive muscle atrophy was found to have occurred for the cSCI subjects at the time of recruitment. In addition, people with SCI can exhibit a slow response to exercise [15] and may have a blunted heart rate response [10]. Fatigue resistance to exercise is compromised because of oxidative type I muscle fibers taking on the properties of glycolytic type II muscle fibers [34]. These considerations affect the capacity of the aerobic systems to undertake and respond to physical activity. This is reflected by the fact that peak oxygen uptake levels recorded in this investigation were much lower than those potentially achievable in the general population, but are comparable to similarly untrained persons during similar forms of exercise.

A limitation of this study was the inability to perform a real-time measurement of the work being performed by the subject during RATTT. An estimate of the total power required to extend the integrated spring system in the RATTT device is given, but we do not know what proportion of this work is performed by the individual and what proportion is performed by the robotic orthoses. Nonetheless, from changes in oxygen uptake, minute ventilation, and heart rate it is clear that some degree of work is being done, because these parameters reflect an increased metabolic demand from the lower-limb muscles as the prescribed exercise is carried out.

\section{CONCLUSIONS}

In this study, we have shown clear trends in acute exercise responses to RATTT in a small and complex case mix of early-stage SCI. Volitional effort led to an increased cardiopulmonary and ventilatory response during RATTT, and these increases were sustained or marginally improved upon with the addition of FES. Changes in minute ventilation, oxygen uptake, heart rate, and RER were each found to be significant for the iSCI subjects. We conclude that a period of training with volitional contribution could potentially improve cardiopulmonary and ventilatory fitness in those with iSCI, and FES-assisted RATTT may be sufficient to attenuate losses in fitness in those persons with cSCI. This evidence is sufficient to warrant further investigation with a larger sample of subjects using a study design that controls for age and time since injury. Future work should focus on initiating FES-assisted RATTT training at the earliest possible time point after SCI and investigate the dose response to a period of training in the $\mathrm{CSCI}$ and iSCI patient populations.

\section{ACKNOWLEDGMENTS}

\section{Author Contributions:}

Design and planning of study and interpretation of results: C. T. D.

Craven, H. Gollee, S. Coupaud, M. A. Purcell, D. B. Allan.

Drafting of manuscript: C. T. D. Craven, H. Gollee, S. Coupaud, M. A. Purcell, D. B. Allan.

Final approval for submission: C. T. D. Craven, H. Gollee, S. Coupaud, M. A. Purcell, D. B. Allan.

Overall clinical support: M. A. Purcell, D. B. Allan.

Data collection: C. T. D. Craven, S. Coupaud.

Engineering developments: C. T. D. Craven, H. Gollee.

Data analysis: C. T. D. Craven.

Financial Disclosures: The authors have declared that no competing interests exist.

Funding/Support: C. T. D. Craven was supported by a James Watt Scholarship. S. Coupaud gratefully acknowledges the Glasgow Research Partnership in Engineering for funding her research post. Institutional Review: The study was approved by the National Health Service United Kingdom South Glasgow and Clyde Local Research Ethics Committee (ref: 08/S0710/66). Each subject provided informed consent prior to participation.

Participant Follow-Up: The authors plan to notify the study subjects of the publication of this article, subject to contact information being available.

\section{REFERENCES}

1. Jacobs PL, Beekhuizen KS. Appraisal of physiological fitness in persons with spinal cord injury. Top Spinal Cord Inj Rehabil. 2005;10(4):32-50. http://dx.doi.org/10.1310/VJ5R-GH2Q-960D-QJLQ

2. Warburton DE, Nicol CW, Bredin SS. Health benefits of physical activity: the evidence. CMAJ. 2006;174(6):801-9. [PMID:16534088] http://dx.doi.org/10.1503/cmaj.051351

3. DeVivo MJ, Stover SL, Black KJ. Prognostic factors for 12-year survival after spinal cord injury. Arch Phys Med Rehabil. 1992;73(2):156-62. [PMID:1543411]

4. Myers J, Lee M, Kiratli J. Cardiovascular disease in spinal cord injury: an overview of prevalence, risk, evaluation, and management. Am J Phys Med Rehabil. 2007;86(2): 142-52. [PMID:17251696] http://dx.doi.org/10.1097/PHM.0b013e31802f0247 
5. Cardenas DD, Hoffman JM, Kirshblum S, McKinley W. Etiology and incidence of rehospitalization after traumatic spinal cord injury: a multicenter analysis. Arch Phys Med Rehabil. 2004;85(11):1757-63. [PMID:15520970] http://dx.doi.org/10.1016/j.apmr.2004.03.016

6. DeVivo MJ, Krause JS, Lammertse DP. Recent trends in mortality and causes of death among persons with spinal cord injury. Arch Phys Med Rehabil. 1999;80(11):1411-19. [PMID:10569435] http://dx.doi.org/10.1016/S0003-9993(99)90252-6

7. Whiteneck GG, Charlifue SW, Frankel HL, Fraser MH, Gardner BP, Gerhart KA, Krishnan KR, Menter RR, Nuseibeh I, Short DJ, Silver JR. Mortality, morbidity, and psychosocial outcomes of persons spinal cord injured more than 20 years ago. Paraplegia. 1992;30(9):617-30.

[PMID:1408338]

http://dx.doi.org/10.1038/sc.1992.124

8. Sekhon LH, Fehlings MG. Epidemiology, demographics, and pathophysiology of acute spinal cord injury. Spine. 2001;26(24 Suppl):S2-12. [PMID:11805601] http://dx.doi.org/10.1097/00007632-200112151-00002

9. Lee SM, Moore AD, Everett ME, Stenger MB, Platts SH. Aerobic exercise deconditioning and countermeasures during bed rest. Aviat Space Environ Med. 2010;81(1):52-63. [PMID:20058738] http://dx.doi.org/10.3357/ASEM.2474.2010

10. Jacobs PL, Nash MS. Exercise recommendations for individuals with spinal cord injury. Sports Med. 2004;34(11): 727-51. [PMID:15456347] http://dx.doi.org/10.2165/00007256-200434110-00003

11. Coutts KD, Rhodes EC, McKenzie DC. Maximal exercise responses of tetraplegics and paraplegics. J Appl Physiol. 1983;55(2):479-82. [PMID:6618941]

12. Jacobs PL, Mahoney ET, Robbins A, Nash M. Hypokinetic circulation in persons with paraplegia. Med Sci Sports Exerc. 2002;34(9):1401-7. [PMID:12218730] http://dx.doi.org/10.1097/00005768-200209000-00001

13. Muraki S, Ehara Y, Yamasaki M. Cardiovascular responses at the onset of passive leg cycle exercise in paraplegics with spinal cord injury. Eur J Appl Physiol. 2000;81(4): 271-74. [PMID:10664084] http://dx.doi.org/10.1007/s004210050042

14. Hooker SP, Figoni SF, Glaser RM, Rodgers MM, Ezenwa BN, Faghri PD. Physiologic responses to prolonged electrically stimulated leg-cycle exercise in the spinal cord injured. Arch Phys Med Rehabil. 1990;71(11):863-69. [PMID:2222153]

15. Barstow TJ, Scremin AM, Mutton DL, Kunkel CF, Cagle TG, Whipp BJ. Gas exchange kinetics during functional electrical stimulation in subjects with spinal cord injury. Med Sci Sports Exerc. 1995;27(9):1284-91.

\section{[PMID:8531627]}

http://dx.doi.org/10.1249/00005768-199509000-00008

16. Burnham RS, May L, Nelson E, Steadward R, Reid DC. Shoulder pain in wheelchair athletes. The role of muscle imbalance. Am J Sports Med. 1993;21(2):238-42.

[PMID:8465919] http://dx.doi.org/10.1177/036354659302100213

17. Dunne AC, Allan DB, Hunt KJ. Characterisation of oxygen uptake response to linearly increasing work rate during robotics-assisted treadmill exercise in incomplete spinal cord injury. Biomed Signal Process Control. 2010;5:70-75. http://dx.doi.org/10.1016/j.bspc.2009.09.003

18. Berry HR, Perret C, Saunders BA, Kakebeeke TH, Donaldson NN, Allan DB, Hunt KJ. Cardiorespiratory and power adaptations to stimulated cycle training in paraplegia. Med Sci Sports Exerc. 2008;40(9):1573-80. [PMID:18685535] http://dx.doi.org/10.1249/MSS.0b013e318176b2f4

19. Hooker SP, Scremin AM, Mutton DL, Kunkel CF, Cagle G. Peak and submaximal physiologic responses following electrical stimulation leg cycle ergometer training. J Rehabil Res Dev. 1995;32(4):361-66. [PMID:8770800]

20. Barstow TJ, Scremin AM, Mutton DL, Kunkel CF, Cagle TG, Whipp BJ. Changes in gas exchange kinetics with training in patients with spinal cord injury. Med Sci Sports Exerc. 1996;28(10):1221-28. [PMID:8897377] http://dx.doi.org/10.1097/00005768-199610000-00003

21. Hooker SP, Figoni SF, Rodgers MM, Glaser RM, Mathews T, Suryaprasad AG, Gupta SC. Physiologic effects of electrical stimulation leg cycle exercise training in spinal cord injured persons. Arch Phys Med Rehabil. 1992;73(5):470-76. [PMID:1580776]

22. Mutton DL, Scremin AM, Barstow TJ, Scott MD, Kunkel $\mathrm{CF}$, Cagle TG. Physiologic responses during functional electrical stimulation leg cycling and hybrid exercise in spinal cord injured subjects. Arch Phys Med Rehabil. 1997;78(7):712-18. [PMID:9228873] http://dx.doi.org/10.1016/S0003-9993(97)90078-2

23. Shibao C, Grijalva CG, Raj SR, Biaggioni I, Griffin MR. Orthostatic hypotension-related hospitalizations in the United States. Am J Med. 2007;120(11):975-80. [PMID:17976425] http://dx.doi.org/10.1016/j.amjmed.2007.05.009

24. Elokda AS, Nielsen DH, Shields RK. Effect of functional neuromuscular stimulation on postural related orthostatic stress in individuals with acute spinal cord injury. J Rehabil Res Dev. 2000;37(5):535-42. [PMID:11322152]

25. Czell D, Schreier R, Rupp R, Eberhard S, Colombo G, Dietz V. Influence of passive leg movements on blood circulation on the tilt table in healthy adults. J Neuroeng Rehabil. 2004;1(1):4. [PMID:15679913] http://dx.doi.org/10.1186/1743-0003-1-4 
26. Chi L, Masani K, Miyatani M, Adam Thrasher T, Wayne Johnston K, Mardimae A, Kessler C, Fisher JA, Popovic MR. Cardiovascular response to functional electrical stimulation and dynamic tilt table therapy to improve orthostatic tolerance. J Electromyogr Kinesiol. 2008;18(6): 900907. [PMID:18835189] http://dx.doi.org/10.1016/j.jelekin.2008.08.007

27. Thrasher T, Keller T, Lawrence M, Popovic M. Effects of isometric FES and dynamic FES on cardiovascular parameters on an active tilt-table stepper. In: Sawan M, editor. Proceedings of the 10th Annual Conference of the International Functional Electrical Stimulation Society; 2005 Jul 5-8; Montreal, Canada. Glendale (CA): IFESS; 2005.

28. Wasserman K, Hansen J, Sue D, Stringer W, Whipp B. Principles of exercise testing and interpretation. Baltimore (MD): Lippincott, Williams and Wilkins; 2005. 585 p.

29. Klabunde RE. Cardiovascular physiology concepts. Baltimore (MD): Lippincott, Williams and Wilkins; 2004. 256 p.

30. Lewington S, Clarke R, Qizilbash N, Peto R, Collins R; Prospective Studies Collaboration. Age-specific relevance of usual blood pressure to vascular mortality: a meta-analysis of individual data for one million adults in 61 prospective studies. Lancet. 2002;360(9349):1903-13. [PMID:12493255] http://dx.doi.org/10.1016/S0140-6736(02)11911-8

31. World Health Organization. Global proceedings on physical activity for health. Geneva (Switzerland): WHO Press; 2010. $60 \mathrm{p}$.

32. Wilmore JH, Costill DL. Physiology of sport and exercise. Champaign (IL): Human Kinetics; 2005.
33. Janssen TW, Dallmeijer AJ, Veeger DJ, van der Woude LH. Normative values and determinants of physical capacity in individuals with spinal cord injury. J Rehabil Res Dev. 2002;39(1):29-39. [PMID:11930906]

34. Salmons S. Adaptive change in electrically stimulated muscle: a framework for the design of clinical protocols. Muscle Nerve. 2009;40(6):918-35. [PMID:19902542] http://dx.doi.org/10.1002/mus.21497

Submitted for publication February 8, 2012. Accepted in revised form August 21, 2012.

This article and any supplemental material should be sited as follows:

Craven CT, Gollee H, Coupaud S, Purcell MA, Allan DB. Investigation of robotic-assisted tilt-table therapy for early-stage spinal cord injury rehabilitation. J Rehabil Res Dev. 2013;50(3):367-78.

http://dx.doi.org/10.1682/JRRD.2012.02.0027

ResearcherID/ORCID: Colm T. D. Craven, MEngSc: A8031-2012; Henrik Gollee, PhD: E-9247-2010; Sylvie Coupaud, PhD: E-9245-2010

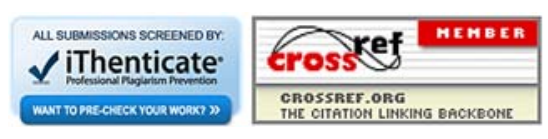


